\title{
Moyamoya Syndrome Associated with Basal Meningioma Successfully Treated by the Modified Transsphenoidal Approach: Case Report
}

\author{
Yoshikazu Ogawa ${ }^{1}$ Miki Fujimura ${ }^{1}$ Teiji Tominaga ${ }^{2}$ \\ ${ }^{1}$ Department of Neurosurgery, Kohnan Hospital, Taihaku-ku, Sendai, \\ Miyagi, Japan \\ 2 Department of Neurosurgery, Tohoku University Graduate School of \\ Address for correspondence Yoshikazu Ogawa, MD, PhD, Department \\ of Neurosurgery, Kohnan Hospital, 4-20-1 Nagamachiminami, Taihaku-ku, \\ Sendai, Miyagi 982-8523, Japan (e-mail: yogawa@kohnan-sendai.or.jp).
} Medicine, Aoba-ku, Sendai, Miyagi, Japan

J Neurol Surg Rep 2014;75:e77-e80.

\begin{abstract}
Keywords

- cerebral ischemia

- extended transsphenoidal approach

- meningioma

- moyamoya disease

- skull base

Objective Moyamoya vasculopathy associated with skull base tumors has been rarely reported except for partially removed pituitary adenoma and craniopharyngioma. Only a single case of meningioma resulted in vast cerebral infarction. Surgical treatment carries a high risk of damage to the compensatory collateral circulation through leptomeningeal anastomosis leading to a devastating outcome.

Case presentation A 46-year-old woman presented to our hospital with progressive visual disturbance. She had a history of medically treated moyamoya vasculopathy. Head magnetic resonance imaging revealed a well-demarcated skull base tumor extending from the planum sphenoidale to the diaphragm sellae. The patient had no cerebral ischemic symptoms, but iodine-123 N-isopropyl-p-iodoamphetamine singlephoton emission computed tomography indicated bilateral hemodynamic compromise. Modified extended transsphenoidal surgery was performed, resulting in successful removal without complications including cerebral ischemia.

Conclusion The very low tolerance to cerebral ischemia associated with moyamoya disease manipulation in this location carries an extremely high risk of cerebral infarction. This is the first report of a basal meningioma associated with moyamoya syndrome that was successfully removed. The modified extended transsphenoidal approach is the firstline surgery for skull base meningioma located in the para-midline region.
\end{abstract}

\section{Introduction}

Moyamoya disease is a chronic and progressive occlusive cerebrovascular disease defined as stenosis of the terminal portion of the bilateral internal carotid arteries (ICAs) and compensatory development of abnormal vascular networks at the base of the brain. Association with brain tumors is excluded from the definition of this disease and defined as kin to moyamoya disease (moyamoya syndrome, quasi-moyamoya disease). ${ }^{1}$ Sev-

received

March 9, 2011

accepted

June 16, 2011

published online

January 16, 2014 eral cases of moyamoya vasculopathy associated with brain tumors have been reported, but most of these cases were associated with tumors in the suboccipital fossa. ${ }^{2-4}$ Association of moyamoya vasculopathy with skull base tumors is rare except with pituitary adenoma and craniopharyngioma ${ }^{5-7}$ partially removed through the classical transsphenoidal approach. A case of sphenoidal ridge meningioma treated by craniotomy resulted in vast cerebral infarction, ${ }^{8}$ illustrating that the very slight tolerance of cerebral ischemia requires extremely delicate
License terms

Stuttgart · New York

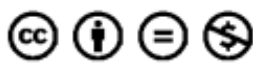


manipulation of the brain and special operative preparations. Fewer cases have been associated with the transcranial approach including Ommaya implantation and partial cystectomy of craniopharyngioma. ${ }^{9,10}$

The transsphenoidal approach has been applied to treat soft tumors originating from the sella turcica. However, recent advances in minimally invasive neurosurgery have developed modified transsphenoidal approaches to treat large and complex lesions beyond the sella turcica, from the crista galli up to and through the odontoid, and laterally to the cavernous sinus. ${ }^{11-13}$ The most important points of this approach are the reduced invasiveness and the approach along the axis of the tumor, avoiding important vessels and nerves with minimal compression of the cerebral cortex. However, the deep and narrow operative field requires extremely delicate dissection and devascularization of the vessels, especially in tough and adherent tumors.

Here we report a case of moyamoya syndrome associated with basal meningioma that was successfully removed through the minimally invasive modified transsphenoidal approach.

\section{Case Presentation}

A 46-year-old woman was introduced to our hospital with aggravating visual acuity in February 2010. She had a history of hypertension since 30 years of age and had been taking medication for diabetes mellitus for 5 years. She had epilepsy, moyamoya vasculopathy was diagnosed, and antiplatelet medication was given at a local hospital at 41 years of age. She had decreased visual acuity in her right eye from around December 2009 that worsened rapidly. Head imaging revealed a skull base tumor compressing the optic chiasm upward, and she was referred to our hospital.

On admission, her consciousness was Japan Coma Scale score 1 , and no neurologic deficit was detected except for the severe visual disturbance. She had only slight light perception in the right eye and temporal hemianopsia in the left eye. Magnetic resonance (MR) imaging showed a well-demarcated skull base tumor extending from the planum sphenoidale to the diaphragm sellae. Abnormal mesh-like enhancement was seen in the right half of the tumor around the right ICA terminal and also at the distal portion of the anterior cerebral artery (ACA) in the convexity (- Fig. 1). MR angiography showed severe stenoses in the bilateral ICA terminals and moyamoya vessels around the horizontal segment of the ACA and the middle cerebral artery (-Fig. 2). Iodine-123 Nisopropyl-p-iodoamphetamine single-photon emission computed tomography using the autoradiographic method indicated that cerebral blood flow and cerebrovascular reactivity were apparently compromised bilaterally (-Fig.3). However, she had not suffered any ischemic symptoms. Her visual acuity deteriorated rapidly. Therefore, tumor removal via extended transsphenoidal surgery was planned.

\section{Operation}

Somatosensory evoked potential (SEP) was monitored by simultaneous stimulation of the left tibial and left median nerves during the operation. $\mathrm{PacO}_{2}$ was maintained at $>40$

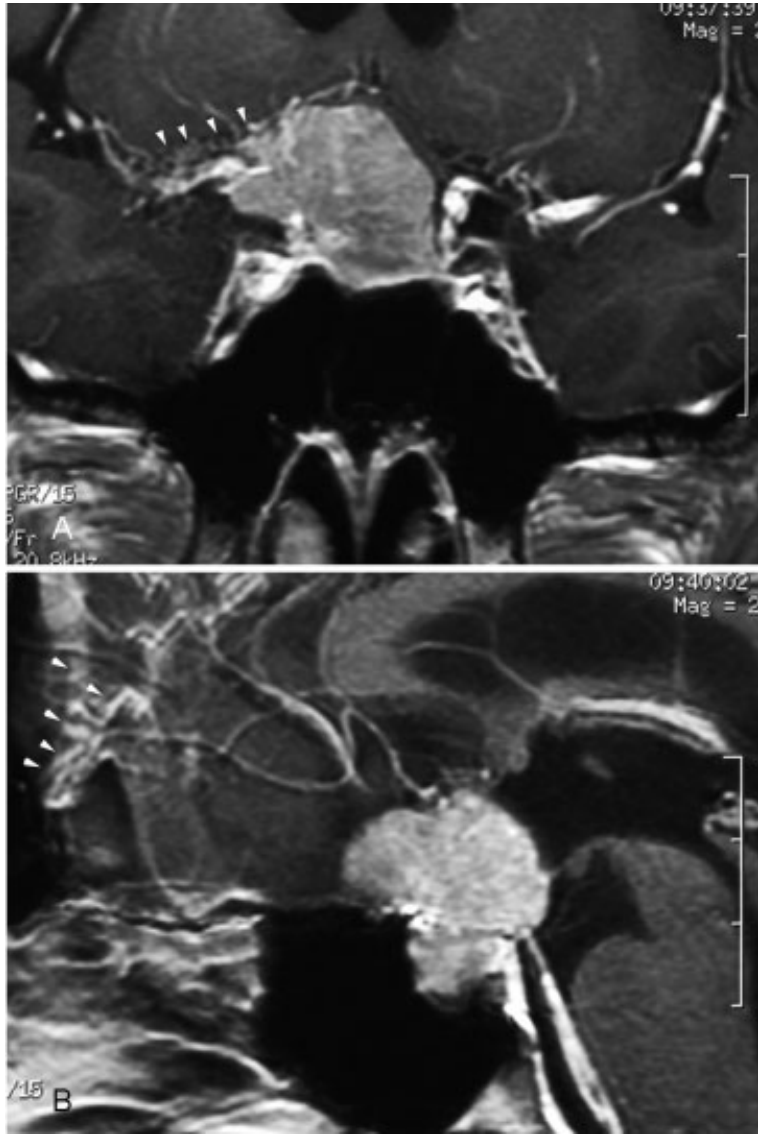

Fig. 1 Head magnetic resonance images with contrast medium showing a well-demarcated skull base tumor compressing the optic chiasm upward and abnormal mesh-like enhancement around the right internal carotid artery terminal and at the distal portion of the anterior cerebral artery (arrowheads). (A) Coronal image. (B) Sagittal image.

$\mathrm{mm} \mathrm{Hg}$, and hematocrit at $>35 \%$ (preoperative hematocrit was 37.4\%). After opening of the sphenoidal and posterior ethmoidal sinuses, the floor of the right optic canal was removed followed by drilling out to a 2 -cm width of the planum sphenoidale to the tuberculum sellae. After adequate coagulation and incision of the dura, the red-ochre elastic

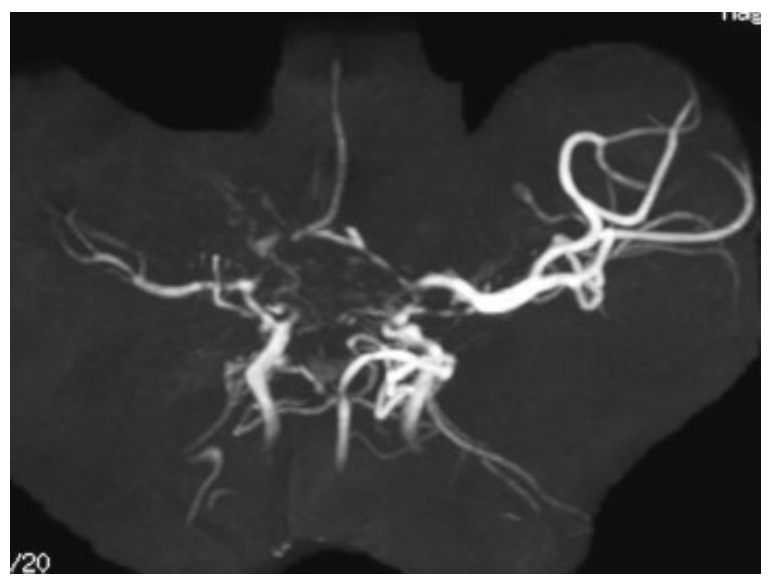

Fig. 2 Head magnetic resonance angiogram revealing severe stenoses of the bilateral internal carotid artery terminals and moyamoya vessels. 

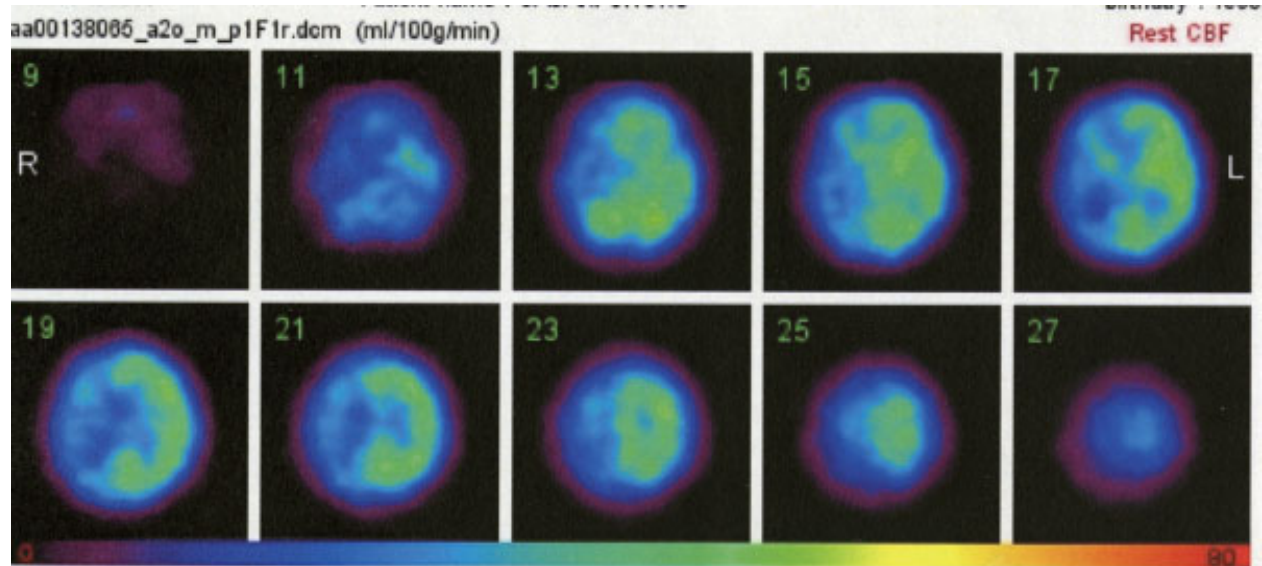

aa00138065_a20_m_p2F2ra.dem (ml/100g/min)
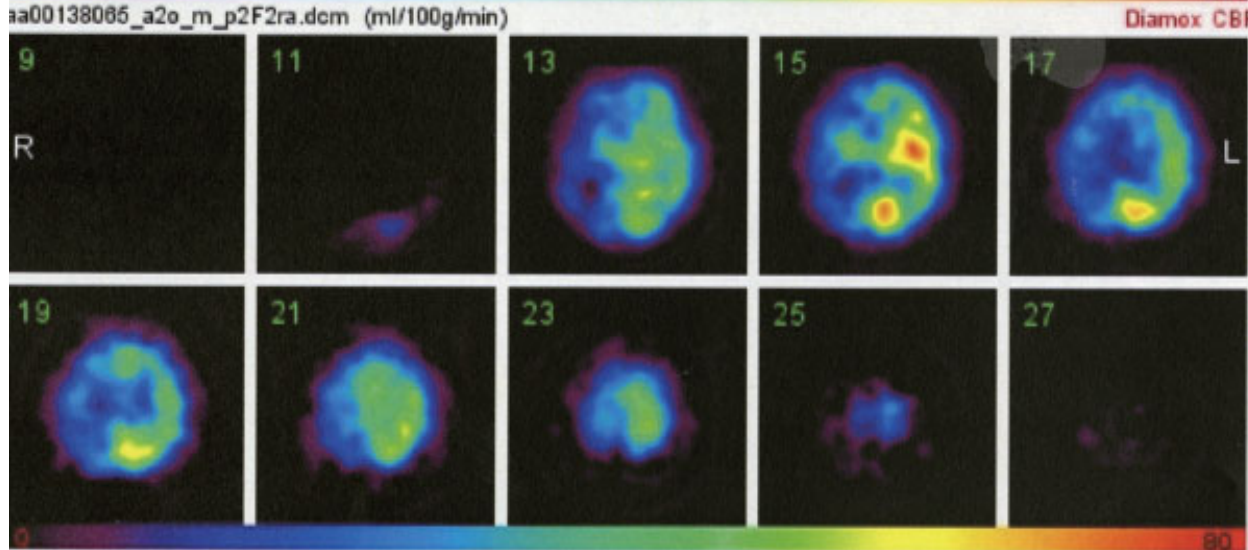

1010/02/24 15:02:30 QSPECT(2.6.1) Java(1.6.0_12)

Fig. 3 lodine-123 N-isopropyl-p-iodoamphetamine single-photon emission computed tomography scans revealing severe reduction of cerebral blood flow, especially in the right hemisphere, and the steal phenomenon bilaterally after injection of acetazolamide.

hard tumor was exposed. The tumor was first detached and fully coagulated from the reverse side of the dura. The tumor bled easily, so it was removed en bloc without internal decompression. The moyamoya vessels were dissected and devascularized at the nearest portion of the tumor surface from the bottom of the right frontal lobe to the anterior edge of the optic chiasm. The encased right ACA was released. The tumor was removed after dissection from the lower plane of the optic chiasm ( - Fig. 4). The SEP remained stable throughout the operation, despite the total operation time of 9.6 hours and the transfusion of $1200 \mathrm{~mL}$ of blood.

\section{Postoperative Course}

The patient woke rapidly from anesthesia. Head MR imaging showed no ischemic lesions on postoperative day 1 , and subsequent MR imaging with contrast medium showed no tumor mass. Her left visual field recovered to normal, but the right eye retained only light perception. The clinical course was uneventful, and she was discharged on postoperative day 13 without any new neurologic deficit.

\section{Discussion}

Moyamoya disease causes the development of compensatory collateral circulation through the posterior communicating arteries and/or posterior pericallosal arteries and also through leptomeningeal anastomosis. Damage to such anastomosis by dural incision in the convexity carries an extremely high risk of ischemic events. Until now only a single case of basal meningioma has been reported by craniotomy. However, the patient had become severely disabled due to vast postoperative cerebral infarction. ${ }^{8}$ In our case, MR imaging clearly showed mesh-like abnormal enhancement at the distal portion of the ACA indicating moyamoya vessels in

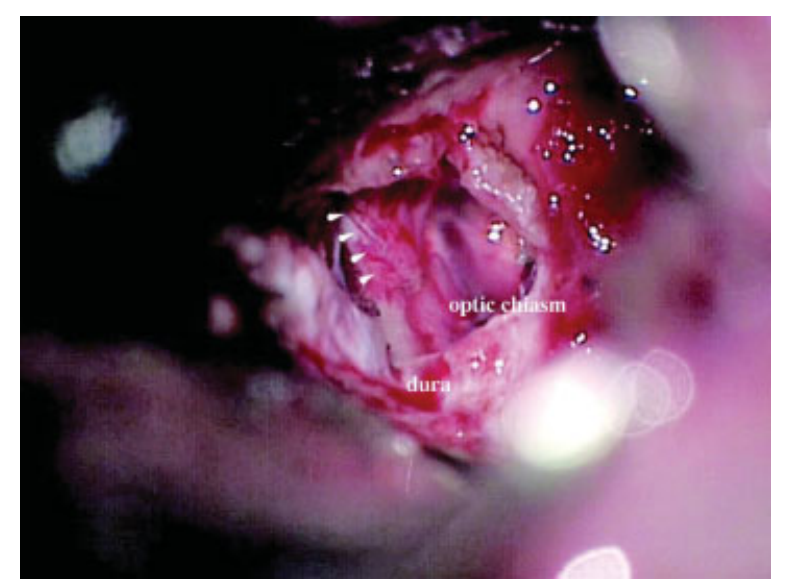

Fig. 4 Intraoperative photograph showing moyamoya vessels on the surface of the atrophic pale optic chiasm. 
the frontal convexity. Avoidance of these moyamoya vessels and compression of the frontal lobe indicated use of the modified transsphenoidal approach.

Although the indications for this approach are limited in the case of midline tumors, the modified extended transsphenoidal approach has great merit for tumor removal. Preservation of cortical blood circulation is always the important principle in every type of neurosurgical operation; maximal care is required, especially in skull base tumors with moyamoya syndrome. Kohno et al emphasized the importance of procedure selection for ruptured basilar artery aneurysm with moyamoya disease in terms of preservation of normal circulation. ${ }^{14}$

\section{Conclusion}

This is the first report of a basal meningioma associated with moyamoya syndrome that was successfully removed through the modified transsphenoidal approach. This approach should be first-line surgery for skull base dural tumors located in the para-midline region.

\section{References}

1 Research on intractable diseases of the Ministry of Health, Labour and Welfare, Japan. Recommendations for the management of moyamoya disease. A statement from Research Committee on Spontaneous Occlusion of the Circle of Willis (Moyamoya Disease). Surg Cerebral Stroke 2009;37:321-337

2 Aihara N, Nagai H, Mase M, Kanai H, Wakabayashi S, Mabe H. Atypical Moyamoya disease associated with brain tumor. Surg Neurol 1992;37(1):46-50

3 Kitano S, Sakamoto H, Fujitani K, Kobayashi Y. Moyamoya disease associated with a brain stem glioma. Childs Nerv Syst 2000;16(4): 251-255
4 Korematsu K, Yoshioka S, Maruyama T, Nagai Y, Tsuji K, Kuratsu J. De novo appearance of cerebellar cavernous malformation in a patient with moyamoya disease: case report and review of the literature. Clin Neurol Neurosurg 2007;109(8):708-712

5 Arita K, Uozumi T, Oki S, et al. Moyamoya disease associated with pituitary adenoma-report of two cases. Neurol Med Chir (Tokyo) 1992;32(10):753-757

6 Kuchiki H, Katakura K, Kinjo T, Sato K, Kayama T. Transsphenoidal surgery and gamma-knife radiosurgery for a treatment of recurrent craniopharyngioma with moyamoya vessels. [in Japanese]. No Shinkei Geka 1998;26(3):273-278

7 Uchida K, Arakawa Y, Ohyama K, et al. Growth hormone-secreting pituitary adenoma associated with primary moyamoya diseasecase report. Neurol Med Chir (Tokyo) 2003;43(7):356-359

8 Kaku M, Nakayama T, Matsukado Y. A case of meningioma accompanied with abnormal vascular network at the base of the brain. Shujutu 1973;27:1159-1162

9 Lau YL, Milligan DW. Atypical presentation of craniopharyngioma associated with Moyamoya disease. J R Soc Med 1986;79(4): 236-237

10 Tsuji N, Kuriyama T, Iwamoto M, Shizuki K. Moyamoya disease associated with craniopharyngioma. Surg Neurol 1984;21(6): 588-592

11 Couldwell WT, Weiss MH, Rabb C, Liu JK, Apfelbaum RI, Fukushima $\mathrm{T}$. Variations on the standard transsphenoidal approach to the sellar region, with emphasis on the extended approaches and parasellar approaches: surgical experience in 105 cases. Neurosurgery 2004;55(3):539-547; discussion 547-550

12 de Divitiis E, Cappabianca P, Cavallo LM. Endoscopic transsphenoidal approach: adaptability of the procedure to different sellar lesions. Neurosurgery 2002;51(3):699-705; discussion 705-707

13 Dusick JR, Esposito F, Kelly DF, et al. The extended direct endonasal transsphenoidal approach for nonadenomatous suprasellar tumors. J Neurosurg 2005;102(5):832-841

14 Kohno K, Abe T, Iwata S, et al. Treatment of ruptured basilar artery aneurysms associated with moyamoya disease. Surg Cerebral Stroke 1996;24:357-364 